\title{
Massive scalar states localized on a de Sitter brane
}

\author{
David Langlois \\ GReCO, Institut d'Astrophysique de Paris (CNRS), \\ 98bis Boulevard Arago, 75014 Paris, France \\ Misao Sasaki \\ Department of Earth and Space Science, \\ Graduate School of Science, \\ Osaka University, Toyonaka 560-0043, Japan
}

\begin{abstract}
We consider a brane scenario with a massive scalar field in the five-dimensional bulk. We study the scalar states that are localized on the brane, which is assumed to be de Sitter. These localized scalar modes are massive in general, their effective four-dimensional mass depending on the mass of the five-dimensional scalar field, on the Hubble parameter in the brane and on the coupling between the brane tension and the bulk scalar field. We then introduce a purely four-dimensional approach based on an effective potential for the projection of the scalar field in the brane, and discuss its regime of validity. Finally, we explore the quasi-localized scalar states, which have a non-zero width that quantifies their probability of tunneling from the brane into the bulk.
\end{abstract}

\section{INTRODUCTION}

A lot of attention has been recently devoted to the braneworld scenario, which provides an alternative to the standard Kaluza-Klein compactification. In fact, this idea was initiated a long time ago by the suggestion [1] that particles could be localized on a defect embedded in a higher-dimensional space, the simplest example being the case of a domain wall, or three-brane, in a five-dimensional bulk spacetime. It was then shown, ignoring gravity, that one could localize scalars and fermions on the domain wall.

Recent progress was achieved on the gravitational aspect of the problem, by showing that it was possible to localize massless gravitons [2], and thus to recover standard four-dimensional gravity, on a brane with tension embedded in an AdS bulk, with the appropriate (negative) cosmological constant $\Lambda=-6 / \ell^{2}$, so that the brane is effectively Minkowski. In addition to the massless gravitons, one finds a continuous spectrum of massive gravitons, starting from zero mass but with a weak coupling to the brane so that their contribution becomes important only on scales of the order of $\ell$ or below.

The analysis of the graviton modes has also been extended to the case of a brane whose effective geometry is de Sitter 3, 4], which is the case when the brane tension more than compensates the cosmological constant. The mode analysis on this background yields a massless graviton, analogous to the four-dimensional graviton, plus a continuum of massive modes, which starts at $(3 / 2) H$. There is thus a gap between the zero mode and the massive modes. A recent work [5] has generalized this treatment to the case of conformally flat cosmological geometries and shown that the minimum gap is $\sqrt{3 / 2} H$.

Not surprisingly, massless bulk scalar fields have properties very similar to the gravitons. In the Randall-Sundrum (RS) background, it is easy to show that, as for gravitons, there exist a localized zero mode and a continuum of arbitrary light states. When one allows for a small but non-vanishing mass, it turns out that the massless bound state disappears and turns into a quasi-localized state with finite mass and finite width, the latter quantifying the probability for the state to decay into the bulk [6].

The purpose of the present work is to study how these properties of massive bulk scalar fields are modified when the brane geometry is de Sitter instead of Minkowski. Massive scalar fields are of special interest for a bulk inflaton model of braneworld inflation [7, 8, 9], where inflation in the brane is driven by a bulk scalar field, in contrast with a brane inflaton model, where inflation is driven by a four-dimensional field confined to the brane [10].

In the present work, we study in detail the bound states of a massive bulk scalar field, localized on a de Sitter brane, taking also into account the possibility of a coupling between the scalar field and the tension of the brane. We show that, depending on the range of values for the scalar field mass $M$ and the coupling, one can find bound states, which can be massless or massive.

We then consider and extend another approach, which consists in determining an effective four-dimensional potential for the projection of the bulk scalar field in the brane, taking into account the five dimensional effects, via the effective four-dimensional Einstein equations. We are thus able to deduce an effective four-dimensional potential that combines the five-dimensional potential and the coupling to the brane. We then compare this approach with the mode approach and find surprisingly good agreement. Namely, given the five-dimensional mass, both the critical coupling allowing for a zero mode and that at which the bound-state ceases to exist agree well with those obtained from the mode 
analysis.

Finally, we also explore some aspects of the quasi-bound states (or quasi-normal modes) in the range of parameters where the bound states no longer exist.

The plan of the paper is the following. In the next section, we present the model and give the equation of motion for the bulk scalar field. In section 3, we discuss the existence of bound states. Section 4 is devoted to an alternative approach based on an effective four-dimensional potential. Finally, we explore, analytically and numerically, the quasi-localized scalar modes, which correspond to modes escaping from the brane into the bulk.

\section{DESCRIPTION OF THE MODEL}

We consider a five-dimensional bulk scalar field $\phi$ and a brane with a tension $\sigma$ coupled to this bulk scalar field. The corresponding action is given by

$$
S=\int d^{5} x \sqrt{-g^{(5)}}\left(\frac{1}{2 \kappa^{2}}\left(R-2 \Lambda_{5}\right)-\frac{1}{2}(\nabla \phi)^{2}-V(\phi)\right)-\int d^{4} x \sqrt{-g^{(4)}} \sigma(\phi),
$$

where we have introduced a five-dimensional (negative) cosmological constant

$$
\Lambda_{5}=-\frac{6}{\ell^{2}}
$$

In this work, we will concentrate, for simplicity, on the case of a quadratic bulk potential,

$$
V(\phi)=\frac{1}{2} M^{2} \phi^{2}
$$

and of a quadratic coupling, i.e. of the form

$$
\sigma(\phi)=\sigma_{0}+\frac{\alpha}{\ell} \phi^{2}
$$

where $\alpha$ is a dimensionless parameter characterizing the 'strength' of the coupling to the brane.

We will take as our background configuration, the solution of the above setup, with the usual cosmological symmetries (homogeneity and isotropy along the three ordinary spatial dimensions), when the scalar field vanishes everywhere. In this case, the bulk is effectively empty of matter and, owing to the cosmological symmetries we impose, its geometry corresponds to $\mathrm{AdS}_{5}$. The metric can be written in the form

$$
d s^{2}=d r^{2}+\ell^{2} \sinh ^{2}(r / \ell)\left[-d \tau^{2}+e^{2 \tau} d \mathbf{x}_{(3)}^{2}\right],
$$

where we have chosen, for simplicity, a flat slicing for the three-dimensional surfaces.

It is possible to insert a brane in such a geometry. Assuming as usual that the bulk is $Z_{2}$ symmetric, i.e. mirror symmetric, about the brane, the junction conditions for the metric at the brane location yield the familiar brane Friedmann equation 11]

$$
H^{2}=\frac{\Lambda_{5}}{6}+\left(\frac{\kappa^{2}}{6}\right)^{2} \sigma_{0}^{2}
$$

In the present case, the Hubble parameter is constant, so that the brane geometry is de Sitter. The Hubble parameter is related to the brane position $r_{0}$, which is fixed in the above coordinate system (2.5), according to the expression

$$
H^{2}=\frac{1}{\ell^{2} \sinh ^{2}\left(r_{0} / \ell\right)}
$$

It is then convenient to rescale the time coordinate so that it corresponds to the cosmic time in the brane. The metric now reads

$$
d s^{2}=d r^{2}+H^{2} \ell^{2} \sinh ^{2}(r / \ell)\left[-d t^{2}+H^{-2} e^{2 H t} d \mathbf{x}_{(3)}^{2}\right] .
$$

It is also convenient to introduce, in addition to the AdS lengthscale $\ell$, another lengthscale, defined from the brane tension $\sigma_{0}$,

$$
\ell_{0}=\left(\frac{\kappa^{2}}{6} \sigma_{0}\right)^{-1}
$$


In the RS case, $\ell=\ell_{0}$, whereas, for a de Sitter brane, $\ell_{0}<\ell$. The brane Hubble parameter is thus given by

$$
H^{2}=\frac{1}{\ell_{0}^{2}}-\frac{1}{\ell^{2}}
$$

Introducing the variable

$$
z=\cosh (r / \ell)
$$

the Hubble parameter given in (2.7) can be reexpressed as

$$
H\left(z_{0}\right)=\frac{1}{\ell \sqrt{z_{0}^{2}-1}}
$$

and the expression of $\ell_{0}$ in terms of $z_{0}$ is therefore

$$
\ell_{0}=\frac{\ell}{z_{0}} \sqrt{z_{0}^{2}-1}
$$

Let us now turn to the scalar field, which will be considered as a test field on the background configuration previously defined. In other words, we will ignore its backreaction on the geometry. In the bulk, the scalar field must satisfy the Klein-Gordon equation, which is given, for the metric (2.8) by

$$
\frac{1}{H^{2} \ell^{2} \sinh ^{2}(r / \ell)} e^{-3 H t} \partial_{t}\left(e^{3 H t} \partial_{t} \phi\right)-\frac{1}{\sinh ^{4}(r / \ell)} \partial_{r}\left(\sinh ^{4}(r / \ell) \partial_{r} \phi\right)+M^{2} \phi=0 .
$$

In addition to this bulk equation, the scalar field must also satisfy the appropriate boundary condition due to the presence of the brane. It can be shown that, for $Z_{2}$ symmetry, this boundary condition is given by

$$
\left.\partial_{r} \phi\right|_{r=r_{0}}=-\frac{1}{2} \frac{d \sigma}{d \phi}=-\frac{\alpha}{\ell} \phi .
$$

It is clear from the two above equations (2.14) and (2.15) that we chose to work with quadratic expressions both for the bulk potential and the brane coupling in order to get linear equations for the scalar field, which greatly simplifies the analysis.

\section{EXISTENCE OF BOUND STATES}

The purpose of this section is to solve explicitly the system of equations 2.14 2.15). Since the bulk equation is separable, it is natural to look for solutions of the form

$$
\phi=u(r) \psi(t),
$$

which leads to a separation of the bulk differential equation (2.14) into a radial equation and a time-dependent equation,

$$
\begin{aligned}
& {\left[\frac{1}{\sinh ^{4}(r / \ell)} \partial_{r}\left(\sinh ^{4}(r / \ell) \partial_{r}\right)-M^{2}+\frac{\lambda^{2}}{\ell^{2} \sinh ^{2}(r / \ell)}\right] u(r)=0,} \\
& {\left[\frac{d^{2}}{d t^{2}}+3 H \frac{d}{d t}+H^{2} \lambda^{2}\right] \psi(t)=0,}
\end{aligned}
$$

where $\lambda^{2}$ is a separation constant. And the boundary condition 2.15) becomes

$$
\frac{d}{d r} u(r)=-\frac{\alpha}{\ell} u(r) \quad \text { at } \quad r=r_{0} .
$$

The solution of the radial equation (3.2) can be expressed in terms of associated Legendre functions. Using the variable $z$ defined in (2.11), one finds

$$
u_{\mu}(z)=\frac{P_{\nu-1 / 2}^{\mu}(z)}{\left(z^{2}-1\right)^{3 / 4}}
$$


with

$$
\mu^{2}=\frac{9}{4}-\lambda^{2}, \quad \nu=\sqrt{M^{2} \ell^{2}+4}
$$

The effective four-dimensional mass of the mode, which appears clearly in the time-dependent equation (3.3), is thus given by

$$
m_{(4)}^{2}=\lambda^{2} H^{2}=\left(9 / 4-\mu^{2}\right) H^{2} .
$$

In the rest of this section, we wish to investigate whether there exist bound state solutions. Bound states modes are defined as solutions which are normalizable, i.e., such that

$$
2 \int_{0}^{r_{0}} d r \sinh ^{2} r u_{\mu}(r) u_{\mu}^{*}(r)=2 \int_{1}^{z_{0}} d z \sqrt{z^{2}-1} u_{\mu}(z) u_{\mu}^{*}(z)<\infty .
$$

Since the behaviour of the Legendre functions when $z$ approaches 1 is given by

$$
P_{\nu}^{\mu}(z) \sim \frac{2^{\mu / 2}}{\Gamma(1-\mu)}(z-1)^{-\mu / 2}, \quad z \rightarrow 1,
$$

the integral in (3.8) converges if $\mu$ is real and negative. Therefore, a bound state exists if one can find a solution (3.5) with $\mu<0$ that also satisfies the boundary condition (3.4), which can be reexpressed in terms of the variable $z$ as

$$
\frac{d}{d z} u+\frac{\alpha}{\left(z^{2}-1\right)^{1 / 2}} u=0 \quad \text { at } z=z_{0} .
$$

Substituting the solution (3.5), this gives the condition

$$
\begin{aligned}
\frac{d}{d z} P_{\nu-1 / 2}^{\mu}-\frac{3}{2} \frac{z}{z^{2}-1} P_{\nu-1 / 2}^{\mu}+\frac{\alpha}{\left(z^{2}-1\right)^{1 / 2}} P_{\nu-1 / 2}^{\mu} & = \\
\frac{1}{z^{2}-1}\left[\left((\nu-2) z+\alpha\left(z^{2}-1\right)^{1 / 2}\right) P_{\nu-1 / 2}^{\mu}-(\mu+\nu-1 / 2) P_{\nu-3 / 2}^{\mu}\right] & =0,
\end{aligned}
$$

where the second expression is obtained by using the recurrence relations satisfied by the associated Legendre functions.

The bound states are delimited by two extreme cases: the massless mode (zero mode), characterized by the value $\mu=-3 / 2$, and the mode at the top of the gap, characterized by the mass-squared $m_{(4)}^{2}=(3 / 2) H^{2}$, i.e., $\mu=0$, above which bound states cannot exist. For each set of values for $H$ (or $z$ ) and $M$, one can thus define two critical values for the coupling, the lower one, $\alpha_{\mathrm{zm}}$, corresponding to a bound state that is also a zero mode and the upper one, $\alpha_{\mathrm{bs}}$ giving the threshold above which bound states disappear.

Using the boundary condition (3.11), one immediately finds that the zero mode coupling, corresponding to $\mu=$ $-3 / 2$, is given by

$$
\alpha_{\mathrm{zm}}(z, M)=-M^{2} \ell^{2} \frac{P_{\nu-1 / 2}^{-5 / 2}}{P_{\nu-1 / 2}^{-3 / 2}} .
$$

where we have used one of the recurrence relations for the Legendre functions and the definition of $\nu$ in terms of $M$. Note that, for $M=0$, one gets $\alpha_{\mathrm{zm}}=0$ : one recovers the usual zero mode of a massless bulk scalar field with no coupling to the brane. As soon as the bulk mass $M$ is nonzero, with $M^{2}>0$, the zero mode coupling becomes negative, which means that one can find a zero mode localized on the brane only with a negative coupling. The case opposite to this occurs for an unstable potential, i.e., $M^{2}<0$ (for $M^{2} \ell^{2}>-4$, which we assume in the present paper).

It is interesting to consider two limits concerning the Hubble parameter of the dS brane: the limit where the Hubble parameter is very small, $H \ell \ll 1$, corresponding to large values of $z$; and, conversely, the limit where $H \ell \gg 1$, corresponding to values of $z$ very close to 1 . For the first limit, one can use the asymptotic behaviour

$$
P_{\nu}^{\mu}(z) \sim 2^{\nu} \pi^{-1 / 2} \frac{\Gamma\left(\nu+\frac{1}{2}\right)}{\Gamma(1+\nu-\mu)} z^{\nu}, \quad z \gg 1,
$$

which leads to the result

$$
\alpha_{\mathrm{zm}} \simeq 2-\nu=2-\sqrt{M^{2} \ell^{2}+4}, \quad H \ell \ll 1 .
$$


For the second limit, the expression (3.9) shows that $\alpha_{\mathrm{zm}}$ behaves like the inverse of the Hubble parameter, more specifically

$$
\alpha_{\mathrm{zm}} \simeq-\frac{M^{2} \ell}{5 H}, \quad H \ell \gg 1 .
$$

Similarly, one can define the largest coupling, $\alpha_{\mathrm{bs}}$, that allows for the existence of a bound state. Using the boundary condition (3.11) with $\mu=0$, it is given by the expression

$$
\alpha_{\mathrm{bs}}(z, M)=\frac{(\nu-1 / 2) P_{\nu-3 / 2}-(\nu-2) z P_{\nu-1 / 2}}{\left(z^{2}-1\right)^{1 / 2} P_{\nu-1 / 2}} .
$$

When the mass of the bulk scalar field vanishes, $M=0$, then $\nu=2$ and the above expression for the critical coupling simplifies and reads

$$
\alpha_{\mathrm{bs}}(z, 0)=\frac{3}{2} \frac{P_{1 / 2}}{\left(z^{2}-1\right)^{1 / 2} P_{3 / 2}} .
$$

As before, it is interesting to consider the behaviour of the maximal coupling in the two limits $z \rightarrow \infty$ and $z \rightarrow 1$. In the first limit, one finds the same result as for $\alpha_{\mathrm{zm}}$, namely

$$
\alpha_{\mathrm{bs}} \simeq 2-\nu=2-\sqrt{M^{2} \ell^{2}+4}, \quad H \ell \ll 1,
$$

which means that a bound state exists only for a value of the coupling very close to $2-\nu$, i.e., negative for $M^{2}>0$ (and positive otherwise). In the second limit, one finds that $\alpha_{\mathrm{bs}}$ is proportional to $H$,

$$
\alpha_{\mathrm{bs}} \simeq \frac{3}{2} H \ell, \quad H \ell \gg 1
$$

In Fig. 1 we have plotted the critical couplings $\alpha_{\mathrm{zm}}$ and $\alpha_{\mathrm{bs}}$ as a function of $z$ for three bulk masses $\left(M^{2} \ell^{2}=1\right.$, $\left.M^{2} \ell^{2}=0, M^{2} \ell^{2}=-1\right)$. This defines, for the three cases, the regions in the coupling parameter space that allow for a bound state. Outside these regions, there is no bound state and the four-dimensional mass of the modes has an imaginary part, as will be discussed later. For large $z$, i.e. for small $H$, the bound state regions become thinner and thinner bands around the line $\alpha=2-\nu$.

Note that, for a positive mass $M$, the critical coupling $\alpha_{\mathrm{bs}}$ is not always positive, but becomes negative for $z>z_{c}(M)$. This means that whereas for $z<z_{c}(M)$, i.e., for a Hubble parameter sufficiently high, one can always find a bound state with a vanishing coupling, it is no longer the case for $z>z_{c}(M)$ where a bound state can be found only with a negative coupling.

Finally, a more general quantitative analysis is possible in the cases where $M^{2} \ell^{2} \ll 1$ and $\alpha \ll 1$. Indeed, by linearizing the boundary condition (3.11) about the zero mode solution characterized by $\mu=-3 / 2, M=0$ and $\alpha=0$, one can find the linear deviation $\Delta \mu=\mu+3 / 2$ as a function of the small parameters $M^{2} \ell^{2}$ and $\alpha$. Expressing $\Delta \mu$ in terms of the effective four-dimensional mass of the bound state, one gets

$$
m_{(4)}^{2} \simeq f_{1}(H \ell) \frac{M^{2}}{2}+f_{2}(H \ell) \frac{2 \alpha}{\ell^{2}},
$$

with

$$
f_{1}(H \ell)=\frac{\sqrt{1+H^{2} \ell^{2}}}{\mathcal{R}(H \ell)}-\frac{3}{2} H^{2} \ell^{2}, \quad f_{2}(H \ell)=\frac{1}{\mathcal{R}(H \ell)}
$$

and

$$
\mathcal{R}(H \ell)=\frac{2 \sqrt{z^{2}-1} P_{1 / 2}^{-3 / 2}(z)}{3 P_{3 / 2}^{-3 / 2}(z)}=\sqrt{1+H^{2} \ell^{2}}-H^{2} \ell^{2} \ln \left(\frac{1+\sqrt{1+H^{2} \ell^{2}}}{H \ell}\right) .
$$

The expression (3.20) shows that, in the linearized limit, the (small) bulk mass-squared and brane coupling contribute additively to the effective four-dimensional mass-squared of the bound state. The coefficients in the linear combination depend on $z$, i.e. on the value of the Hubble parameter. In the limit $H \ell \rightarrow 0$, i.e., $z \rightarrow+\infty$, one finds

$$
m_{(4)}^{2} \simeq \frac{M^{2}}{2}+2 \frac{\alpha}{\ell^{2}}, \quad H \ell \ll 1
$$




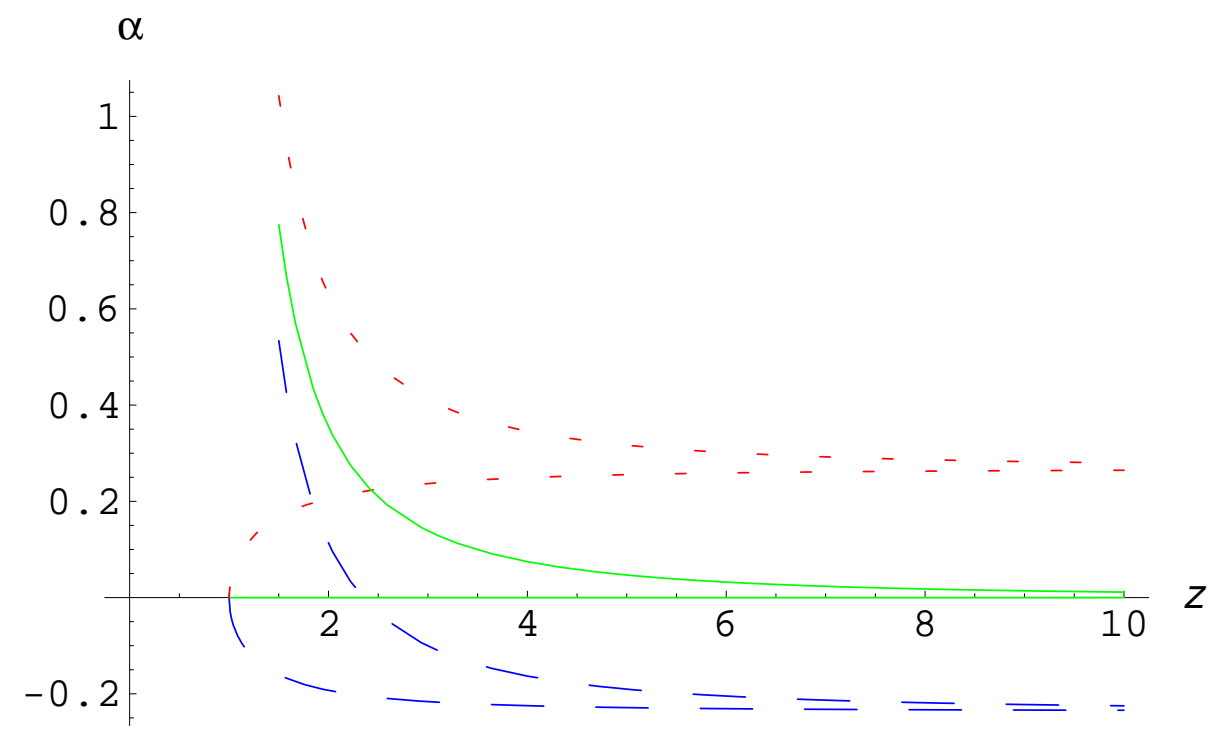

FIG. 1: Range of coupling values $\alpha$ that allow for the existence of a bound state, as a function of $z$ (and thus of $H \ell$ ) for the three following cases: $M^{2} \ell^{2}=1$ (long-dashed blue lines); $M^{2} \ell^{2}=-1$ (short-dashed red lines); $M^{2} \ell^{2}=0$ (continuous green lines). In each case the upper limit corresponds to $\alpha_{\mathrm{bs}}(z, M)$ and the lower limit to $\alpha_{\mathrm{zm}}(z, M)$, both values converging towards $\alpha=2-\sqrt{4+M^{2} \ell^{2}}$ at large $z$.

whereas in the opposite limit one gets

$$
m_{(4)}^{2} \simeq \frac{3}{5} M^{2}+3 \frac{H}{\ell} \alpha, \quad H \ell \gg 1 .
$$

The contribution from the coupling is thus proportional to the coupling parameter $m$ times the largest of the two mass scales $H$ and $\ell^{-1}$. The contribution from the bulk scalar field mass is essentially the same with a very small variation of the coefficient. Note also that the result (3.15) follows immediately from (3.24) with $m_{(4)}^{2}=0$.

In the case of $H \ell \ll 1$, it may be worth mentioning that the coupling $(\alpha / \ell) \phi^{2}$ on the brane contributes to the effective mass term as it is, if we rescale $\phi$ as $\phi \rightarrow \Phi=\sqrt{\ell} \phi$ by introducing an effective four-dimensional scalar field $\Phi$ of correct dimensions, whereas the bulk mass-squared $M^{2}$ contributes with a factor of $1 / 2$.

\section{EFFECTIVE POTENTIAL APPROACH}

In this section, we will use the four-dimensional projection of the five-dimensional Einstein equations onto the brane in order to establish an effective potential for the four-dimensional projection of the scalar field on the brane. Such a procedure was successfully applied in the case of a bulk scalar field with quadratic potential but without coupling to the brane [7, [8] and it was shown that the effective potential is simply half the bulk potential when $H \ell \ll 1$. The purpose of this section is to generalize this result to include the coupling of the scalar field to the brane.

\section{A. General derivation}

We will start by recalling some results derived in [12] following the procedure of [13]. It was shown there in particular that the effective four-dimensional Einstein equations for dilaton-vacuum configurations are given by

$$
{ }^{(4)} G_{\mu \nu}=\frac{2 \kappa_{5}^{2}}{3} \hat{T}_{\mu \nu}(\phi)-{ }^{(4)} \Lambda g_{\mu \nu}-E_{\mu \nu},
$$

with

$$
\hat{T}_{\mu \nu}=D_{\mu} \phi D_{\nu} \phi-\frac{5}{8} g_{\mu \nu}(D \phi)^{2}
$$




$$
{ }^{(4)} \Lambda=\frac{{ }^{(5)} \Lambda}{2}+\frac{1}{2} \kappa_{5}^{2}\left[V(\phi)+\frac{1}{6} \kappa_{5}^{2} \sigma^{2}-\frac{1}{8}\left(\frac{d \sigma}{d \phi}\right)^{2}\right],
$$

where $D_{\mu}$ denotes the covariant differentiation with respect to the metric on the brane. Using the four-dimensional Bianchi identities, the covariant differentiation of Einstein's equations (4.1) implies

$$
D^{\mu} E_{\mu \nu}=\frac{2}{3} \kappa_{5}^{2} D^{\mu} \hat{T}_{\mu \nu}-D_{\nu}^{(4)} \Lambda \text {. }
$$

Specializing to a FLRW (Friedmann-Lemaitre-Robertson-Walker) geometry, one finds that the Friedmann equation on the brane, corresponding to the component (0-0) of (4.1) is given by

$$
3 H^{2}=-\frac{3}{\ell^{2}}+\kappa_{5}^{2}\left(\frac{1}{4} \dot{\phi}^{2}+\frac{1}{2} V+\frac{\kappa_{5}^{2}}{12} \sigma^{2}-\frac{1}{16} \sigma^{\prime 2}\right)+E,
$$

where $E=E_{0}^{0}=-E_{00}$. Equation (4.4) yields

$$
\begin{aligned}
\dot{E}+4 H E & =\kappa_{5}^{2}\left[-\frac{1}{2} \ddot{\phi}-2 H \dot{\phi}-\frac{1}{2} V^{\prime}-\frac{\kappa_{5}^{2}}{12}\left(\sigma^{2}\right)^{\prime}+\frac{1}{16}\left(\sigma^{\prime 2}\right)^{\prime}\right] \dot{\phi} \\
& =\kappa_{5}^{2}\left[\frac{1}{2}(\ddot{\phi}+2 H \dot{\phi})-(\ddot{\phi}+3 H \dot{\phi})-\frac{1}{2} V^{\prime}-\frac{\kappa_{5}^{2}}{12}\left(\sigma^{2}\right)^{\prime}+\frac{1}{16}\left(\sigma^{\prime 2}\right)^{\prime}\right] \dot{\phi},
\end{aligned}
$$

where we have rewritten the terms in the brackets involving the first and second derivatives of $\phi$, as two linear combinations, one which will be easily integrated and the other one corresponding to the familiar four-dimensional Klein-Gordon equation.

We are now going to assume that the brane value of the scalar field satisfies a Klein-Gordon equation that can be written in the form

$$
\ddot{\phi}+3 H \dot{\phi}+V_{\text {eff }}^{\prime}=-J
$$

where $V_{\text {eff }}$ is an effective potential which we wish to determine and where $J$ stands for a possible energy leak out of the brane into the bulk. Substituting this Klein-Gordon equation in Eq. (4.6) above, one finds

$$
\dot{E}+4 H E=\frac{\kappa_{5}^{2}}{2}(\ddot{\phi}+2 H \dot{\phi}) \dot{\phi}+\kappa_{5}^{2}\left[V_{\text {eff }}^{\prime}-\frac{1}{2} V^{\prime}-\frac{\kappa_{5}^{2}}{12}\left(\sigma^{2}\right)^{\prime}+\frac{1}{16}\left(\sigma^{\prime 2}\right)^{\prime}+J\right] \dot{\phi} .
$$

This strongly suggests that the effective potential, if it makes sense, is of the form

$$
V_{\text {eff }}=-\frac{3}{\kappa_{5}^{2} \ell^{2}}+\frac{1}{2} V+\frac{\kappa_{5}^{2}}{12} \sigma^{2}-\frac{1}{16} \sigma^{\prime 2}
$$

where the choice of the constant is for convenience as will be seen very soon. Remarkably, this is the same combination which appears in the Friedmann equation (4.5). That is, if we adopt this definition for the effective potential, and introduce the quantity $X$ defined by

$$
E=\frac{\kappa_{5}^{2}}{4} \dot{\phi}^{2}+\kappa_{5}^{2} X
$$

the Friedmann equation (4.5) and the equation (4.8) for $E$ reduce to the very simple system

$$
\begin{aligned}
3 H^{2} & =\kappa_{5}^{2}\left[\frac{1}{2} \dot{\phi}^{2}+V_{\mathrm{eff}}+X\right], \\
\dot{X}+4 H X & =J \dot{\phi},
\end{aligned}
$$

where one recognizes the standard four-dimensional Friedmann equation with a scalar field and some extra component $X$. The second equation is a (non)-conservation equation for the extra-component $X$. Note that $X$ plays the role of the Weyl, or dark radiation, which was identified in the simplest model of brane cosmology [11]. When the energy outflow is zero, i.e. $J=0$, one recovers the result

$$
X=\frac{\mathcal{C}}{a^{4}} .
$$

A non zero $J$ means that there is an energy outflow from the brane into the bulk, which is going to feed the Weyl energy density. This is similar somehow to the growth of $\mathcal{C}$ induced by the gravitational wave emission from brane cosmological perturbations, which was recently analysed in [14]. It should also be mentioned that we have not really proved the form (4.9) for the effective potential. We have simply shown that this form is consistent with the system of effective equations in the brane. 


\section{B. Exact solutions}

It is instructive at this stage to check the above approach for known exact solutions. One of the simplest examples is the case of a bulk scalar field with the exponential potential [15, 16, 17, 18, 19]

$$
V(\phi)=V_{0} \exp \left(-\frac{2}{\sqrt{3}} \lambda \kappa \phi\right) .
$$

One can find for the bulk with vanishing cosmological constant explicit static solutions, which read

$$
d s^{2}=-h(R) d T^{2}+\frac{R^{2 \lambda^{2}}}{h(R)} d R^{2}+R^{2} d \vec{x}^{2},
$$

for the metric, with

$$
h(R)=-\frac{\kappa^{2} V_{0} / 6}{1-\left(\lambda^{2} / 4\right)} R^{2}-\mathcal{C} R^{\lambda^{2}-2}
$$

$\mathcal{C}$ being an arbitrary constant, and

$$
\frac{\kappa}{\sqrt{3}} \phi=\lambda \ln (R)
$$

for the scalar field.

A brane with a tension

$$
\sigma(\phi)=\sigma_{0} \exp \left(-\frac{\lambda}{\sqrt{3}} \kappa \phi\right)
$$

will undergo a cosmological evolution governed by the generalized Friedmann equation

$$
H^{2}=\left[\frac{\kappa^{4}}{36} \sigma_{0}^{2}+\frac{\kappa^{2} V_{0} / 6}{1-\left(\lambda^{2} / 4\right)}\right] R^{-2 \lambda^{2}}+\mathcal{C} R^{-4-\lambda^{2}}
$$

Since the bulk cosmological constant is zero, the effective potential is here

$$
\begin{aligned}
V_{\text {eff }}=\frac{1}{2} V+\frac{\kappa_{5}^{2}}{12} \sigma^{2}-\frac{1}{16} \sigma^{\prime 2} & =\left[\frac{V_{0}}{2}+\frac{\kappa^{2}}{12}\left(1-\frac{\lambda^{2}}{4}\right) \sigma_{0}^{2}\right] \exp \left(-\frac{2}{\sqrt{3}} \lambda \kappa \phi\right) \\
& \equiv V_{\text {eff }, 0} \exp \left(-\frac{2}{\sqrt{3}} \lambda \kappa \phi\right)
\end{aligned}
$$

It is not difficult to check that $\phi$ satisfies the effective Klein-Gordon equation (4.7) with

$$
J=-\left(1-\frac{\lambda^{2}}{2}\right) H \dot{\phi} .
$$

The Friedmann equation (4.19) can also be written in the effective form (4.11) with the energy density $X$ given by

$$
\begin{aligned}
\kappa^{2} X & =3\left(1-\frac{\lambda^{2}}{4}\right) \mathcal{C} R^{-4-\lambda^{2}}-\frac{\kappa^{2}}{4} \dot{\phi}^{2} \\
& =3\left(1-\frac{\lambda^{2}}{2}\right) \mathcal{C} R^{-4-\lambda^{2}}-\frac{\lambda^{2} / 4}{1-\lambda^{2} / 4} V_{\text {eff }, 0} R^{-2 \lambda^{2}} .
\end{aligned}
$$

Thus, if we apply our interpretation of $J$ that it describes energy flow from the brane into the bulk, the above analysis implies that the energy is actually flowing onto the brane for $\lambda^{2}<2$ rather than flowing out of the brane. It is also worth noting that, whereas the present exemple is an instructive check, it is however not very useful in practice because the energy exchange between the brane and the bulk is important so that the choice between the variables $X$ and $E$ is somewhat arbitrary (in fact, the expression for $E$ in terms of the scale factor is simpler). It is really for the cases where the energy leak $J$ is small that the effective potential approach makes really sense physically. 


\section{Quadratic case}

In the case of the quadratic bulk potential and brane coupling, 2.32.4, the effective potential suggested by the above analysis reads (with Eq. (2.9) in mind),

$$
V_{\mathrm{eff}}=\frac{3}{\kappa_{5}^{2}}\left(\frac{1}{\ell_{0}^{2}}-\frac{1}{\ell^{2}}\right)+\frac{1}{2}\left(\frac{1}{2} M^{2}+2 \frac{\alpha}{\ell \ell_{0}}-\frac{\alpha^{2}}{2 \ell^{2}}\right) \phi^{2}+\frac{\kappa_{5}^{2}}{12} \frac{\alpha^{2}}{\ell^{2}} \phi^{4} .
$$

At the extremum $\phi=0$, the effective mass-squared is thus given by

$$
\mathcal{M}_{\text {eff }}^{2}=\frac{1}{2} M^{2}+2 \frac{\alpha}{\ell^{2}} \sqrt{1+H^{2} \ell^{2}}-\frac{\alpha^{2}}{2 \ell^{2}}
$$

where we have replaced $\ell_{0}$ by its expression in terms of $\ell$ and $H$. It is interesting to note that this potential, which is quartic in $\phi$, takes a double-well form for $\mathcal{M}_{\text {eff }}^{2}<0$. If the present approach is valid, this implies that we may describe a situation of spontaneous symmetry breaking with $V_{\text {eff }}$.

We now wish to compare the results of the mode analysis with the effective potential approach, and in order to so, to compare the effective mass predicted by the two analyses. In the previous section, we have identified two critical cases: the case when the bound state is a zero mode, i.e., $m_{(4)}^{2}=0$, and the case when the bound state reaches the top of the gap, i.e., $m_{(4)}^{2}=(9 / 4) H^{2}$. In the effective potential approach, the equation

$$
\mathcal{M}_{\text {eff }}^{2} \equiv \frac{2 \alpha}{\ell \ell_{0}}-\frac{\alpha^{2}}{2 \ell^{2}}+\frac{M^{2}}{2}=0
$$

is easily solve to yield

$$
\hat{\alpha}_{\mathrm{zm}}(z, M)=\frac{2 z}{\sqrt{z^{2}-1}} \pm \sqrt{\frac{4 z^{2}}{z^{2}-1}+M^{2} \ell^{2}} .
$$

Similarly, the bound state threshold is determined by solving the equation

$$
\mathcal{M}_{\text {eff }}^{2} \equiv \frac{2 \alpha}{\ell \ell_{0}}-\frac{\alpha^{2}}{2 \ell^{2}}+\frac{M^{2}}{2}=\frac{9 / 4}{\ell^{2}\left(z^{2}-1\right)},
$$

which gives

$$
\hat{\alpha}_{\mathrm{bs}}(z, M)=\frac{2 z}{\sqrt{z^{2}-1}} \pm \sqrt{\frac{4 z^{2}}{z^{2}-1}+M^{2} \ell^{2}-\frac{9 / 2}{\left(z^{2}-1\right)}} .
$$

In both cases, we will keep only the root with the minus sign since it matches with the small coupling limit. In the small Hubble parameter limit, i.e., in the large $z$ limit, the two expressions converge towards the same value $2-\nu$ as found previously.

For small bulk mass and coupling, the effective potential approach can provide a reasonable approximation, even in the case $H \ell \gg 1$. Indeed, neglecting the $\alpha^{2}$ term in (4.24), one gets

$$
\mathcal{M}_{\mathrm{eff}}^{2} \simeq \frac{1}{2} M^{2}+2 \frac{\alpha}{\ell^{2}} \sqrt{1+H^{2} \ell^{2}}, \quad(\alpha \ll M \ell) .
$$

Therefore, in the limit $H \ell \ll 1$, one finds

$$
\mathcal{M}_{\text {eff }}^{2} \simeq \frac{1}{2} M^{2}+2 \frac{\alpha}{\ell^{2}}, \quad H \ell \ll 1,
$$

which is exactly the same result as in (3.23). This strongly indicates the validity of the effective potential approach for $H \ell \ll 1$, at least in the case of quadratic potential and brane-coupling. It is then tempting to conjecture that this approach is valid for more general cases, including the case when the backreaction of the scalar field dynamics to the geometry is non-negligible.

Even in the opposite limit $H \ell \gg 1$, one finds

$$
\mathcal{M}_{\text {eff }}^{2} \simeq \frac{1}{2} M^{2}+2 \frac{H}{\ell} \alpha, \quad H \ell \gg 1,
$$

which is qualitatively similar to (3.24) although the numerical coefficients in the linear combination are now slightly different. 


\section{QUASI-NORMAL MODES}

We have so far concentrated our attention on stable, bound-state modes. It is however instructive to study as well the decaying, quasi-normal modes in the case where there exists no bound-state mode, although this is a more complicated problem. In particular, if the effective potential we derived in the previous section is valid, the term $J$ which describes the possible energy leak to the bulk may be determined by studying the decay width of quasi-normal modes.

Mathematically, quasi-normal modes are defined as those that satisfy the purely outgoing-wave boundary condition at future Cauchy horizon of $\mathrm{AdS}_{5}$. A quasi-normal mode can be found by solving Eq. (3.11) for complex $\mu$ with $\operatorname{Re}[\mu]>0$, with $\operatorname{Im}\left[\mu^{2}\right]$ being related to the decay width of the effective four-dimensional mass $m_{(4)}$. Note that, if $\mu$ is a solution to Eq. (3.11), so is its complex conjugate $\mu^{*}$. For definiteness, let us reserve the terminology 'quasibound-state modes' for those modes with non-zero imaginary part, and call the modes with positive real $\mu$ the 'purely decaying modes'.

\section{A. Analytical approach}

In the limit $H^{2} \ell^{2} \ll 1$, one can obtain an analytic expression for the quasi-bound-state modes with least real part, i.e., the effective mass with smallest decay width. In this case, it is convenient to use one of the decompositions of the associated Legendre function into hypergeometric functions [20]:

$$
P_{\nu}^{\mu}(z)=A_{1} F\left(\frac{1}{2}+\frac{\nu}{2}-\frac{\mu}{2}, \frac{1}{2}+\frac{\nu}{2}+\frac{\mu}{2} ; \nu+\frac{3}{2}, \frac{1}{1-z^{2}}\right)+A_{2} F\left(-\frac{\nu}{2}+\frac{\mu}{2},-\frac{\nu}{2}-\frac{\mu}{2} ;-\nu+\frac{1}{2}, \frac{1}{1-z^{2}}\right),
$$

with

$$
A_{1}=\frac{2^{-\nu-1} \Gamma\left(-\frac{1}{2}-\nu\right)}{\sqrt{\pi} \Gamma(-\nu-\mu)}\left(z^{2}-1\right)^{-(\nu+1) / 2}, \quad A_{2}=\frac{2^{\nu} \Gamma\left(\frac{1}{2}+\nu\right)}{\sqrt{\pi} \Gamma(1+\nu-\mu)}\left(z^{2}-1\right)^{\nu / 2} .
$$

Substituting this expression into the junction condition (3.11), and using $z^{2}-1=(H \ell)^{-2}$, one finds, by expanding in terms of $H^{2} \ell^{2}$,

$$
\begin{aligned}
(\nu-2+\alpha)(\nu-1) & +\left\{\frac{1}{2}(\nu-2)(\nu-1)+\frac{1}{4}\left[\left(\frac{1}{2}-\nu\right)^{2}-\mu^{2}\right](\nu-4+\alpha)\right\} H^{2} \ell^{2}+\mathcal{O}\left(H^{4} \ell^{4}\right) \\
& +2^{-2 \nu} \frac{\Gamma(-\nu) \Gamma\left(\frac{1}{2}+\nu-\mu\right)}{\Gamma(\nu) \Gamma\left(\frac{1}{2}-\nu-\mu\right)}(1-\nu)(\nu+2-\alpha)\left(H^{2} \ell^{2}\right)^{\nu}+\mathcal{O}\left(\left(H^{2} \ell^{2}\right)^{\nu+2}\right)=0 .
\end{aligned}
$$

We stress that this expansion makes sense only for $\mu^{2} H^{2} \ell^{2} \ll 1$. The dominant terms are the first two terms which give

$$
\mu^{2} H^{2} \ell^{2} \simeq\left(\frac{1}{2}-\nu\right)^{2} H^{2} \ell^{2}+4 \frac{(\nu-2+\alpha)(\nu-1)}{\nu-4+\alpha}
$$

For this equation to be consistent with the condition $\mu^{2} H^{2} \ell^{2} \ll 1$, one must assume $\nu-2+\alpha \ll 1$, which means that one must be very close to the bound state region defined before. In this case, the solutions are

$$
\mu \simeq \pm \sqrt{\left(\alpha-\frac{3}{2}\right)^{2}-2 \frac{(1-\alpha)(\nu-2+\alpha)}{H^{2} \ell^{2}}} .
$$

In the more particular case where both $M^{2} \ell^{2} \ll 1$ and $\alpha \ll 1$, one finds

$$
\mu \simeq \pm \sqrt{\frac{9}{4}-\frac{M^{2} \ell^{2}+4 \alpha}{2 H^{2} \ell^{2}}} .
$$

It may be worth mentioning that this result is valid irrespective of the relative magnitudes of $H \ell, M \ell$ and $\alpha$, as long as they are all small compared to unity. In particular, if the quantity inside the square root is positive, the solution with the minus sign (i.e., $\mu<0$ ) is just a bound-state solution in the limit $H \ell \ll 1$, given by Eq. (3.23). On the other hand, if the quantity inside the square root is negative, the dominant term of $\mu$ will become purely imaginary and a 
small real part will develop in $\mu$, as a result of the appearance of an imaginary part in $\mu^{2}$. The imaginary part of $\mu^{2}$, however, cannot be determined from the first line of (5.3) which contains only polynomial functions of $\mu^{2}$. One must resort to the second line to compute its imaginary part, which will be given by

$$
H^{2} \ell^{2} \operatorname{Im}\left[\mu^{2}\right] \simeq \frac{2^{2-2 \nu}(1-\nu)(\nu+2-\alpha)(H \ell)^{2 \nu}}{(\nu-4+\alpha)} \operatorname{Im}\left[\frac{\Gamma(-\nu) \Gamma\left(\frac{1}{2}+\nu-\mu\right)}{\Gamma(\nu) \Gamma\left(\frac{1}{2}-\nu-\mu\right)}\right],
$$

where one can substitute the solution (5.5) in the right hand side. This expression agrees with [7] for $\alpha=0$.

In the limit $H^{2} \ell^{2} \ll M^{2} \ell^{2} \ll 1$ and $H^{2} \ell^{2} \ll \alpha \ll 1$, one finds

$$
H^{2} \ell^{2} \operatorname{Im}\left[\mu^{2}\right] \simeq \pm \frac{\pi}{16}\left(M^{2} \ell^{2}+4 \alpha\right)^{2}
$$

which agrees with the Minkowski limit of [6] in the case $\alpha=0$.

Another case which may be studied analytically, though only qualitatively, is when $\alpha \gg 1$. In this case Eq. (3.11) implies that the terms proportional to $\alpha$ should become $O(1 / \alpha)$. Hence, in the limit $\alpha \rightarrow \infty, \mu$ is given by a zero of $P_{\nu-1 / 2}^{\mu}(z)$ on the complex $\mu$-plane (with $\operatorname{Re}[\mu]>0$ and $\operatorname{Im}[\mu] \neq 0$ ), which is independent of $\alpha$. Thus $\mu$ converges to a finite value in the limit $\alpha \rightarrow \infty$.

Let us next discuss the purely decaying modes for which $\mu$ lies on the (positive) real axis, i.e., with no real part in the effective mass. They are usually subdominant in the sense that their decay widths are larger than the decay width of the complex quasi-bound-state modes discussed above. For $H \ell \ll 1$, the asymptotic behaviour of Legendre functions is given in (3.13) and one thus finds the zeros of the boundary condition (3.11) when the common dominator $\Gamma(1-\mu)$ for all terms goes to infinity, i.e. for

$$
\mu_{n}=1+n, \quad n=0,1,2, \ldots
$$

The other limit is $H \ell \gg 1$ and the Legendre functions behave according to (3.9). In the boundary condition (3.11), the dominant terms, when $z \gg 1$ are the terms proportional to $P_{\nu-1 / 2}^{\mu}$ and therefore the zeros of (3.11) are given by

$$
\mu_{n}=\frac{1}{2}+\sqrt{4+M^{2}}+n, \quad n=0,1,2 \ldots
$$

where $n$ is a positive integer, corresponding to cases where the Gamma function in the denominator of $P_{\nu-1 / 2}^{\mu}$ blows up. These zeros thus now depend on $M$ but not on the coupling $\alpha$ or on $z$ (provided however $z \gg 1$ ).

\section{B. Numerical approach}

In order to study the quasi-normal modes in more details, we have solved numerically the following equation for the complex number $\mu$,

$$
F(\mu ; M, \alpha, z) \equiv-(\mu+\nu-1 / 2) \frac{P_{\nu-3 / 2}^{\mu}}{P_{\nu-1 / 2}^{\mu}}+(\nu-2) z+\alpha\left(z^{2}-1\right)^{1 / 2}=0,
$$

for various values of the parameters $M, \alpha$ and $z$. As mentioned earlier, if $\mu$ is a solution, so is $\mu^{*}$. Hence, without loss of generality, we may confine our search for $\mu$ on the first quadrant (i.e., $\operatorname{Re}[\mu]>0$ and $\operatorname{Im}[\mu]>0)$ on the complex $\mu$-plane.

Let us start our numerical exploration with the case of a vanishing coupling $\alpha=0$. In Fig. 2 we have plotted the numerical solutions for $\mu$ in the complex plane for various values of the bulk mass $M$. As can be seen in the figure, we have obtained several branches of solutions which evolve continously as $M \ell$ varies. As $M \ell$ increases, the modes on these branches migrate away from the real axis. The branch closest to the vertical axis is the most important dynamically because its imaginary part is small.

This branch is connected to the origin of the complex plane in the limit $M \ell \rightarrow 0$ and the corresponding quasinormal modes have been computed analytically just above for $H \ell \ll 1$ and $M^{2} \ell^{2} \ll 1$. As a check, we can compare the analytical results given above with our numerical solutions. As noted before, we perform our search in the first quadrant of the complex $\mu$-plane, which means $\operatorname{Im}\left[m_{(4)}^{2}\right]<0$. First, we have checked that the real part of $m_{(4)}^{2}$ is indeed very close to the analytical value $M^{2} / 2$. For the imaginary part, we have plotted in $\operatorname{Fig}$. [3] $-\operatorname{Im}\left[m_{(4)}^{2}\right] /\left(M^{4} \ell^{2}\right)$ as a function of $M \ell$ in two cases, $z=50$ and $z=1000$. In both cases, starting from high values of $M \ell$, one approaches the analytical value of $\pi / 16$ as $M \ell$ decreases, as suggested by (5.8). But, whereas in the case $z=1000$, the dots 


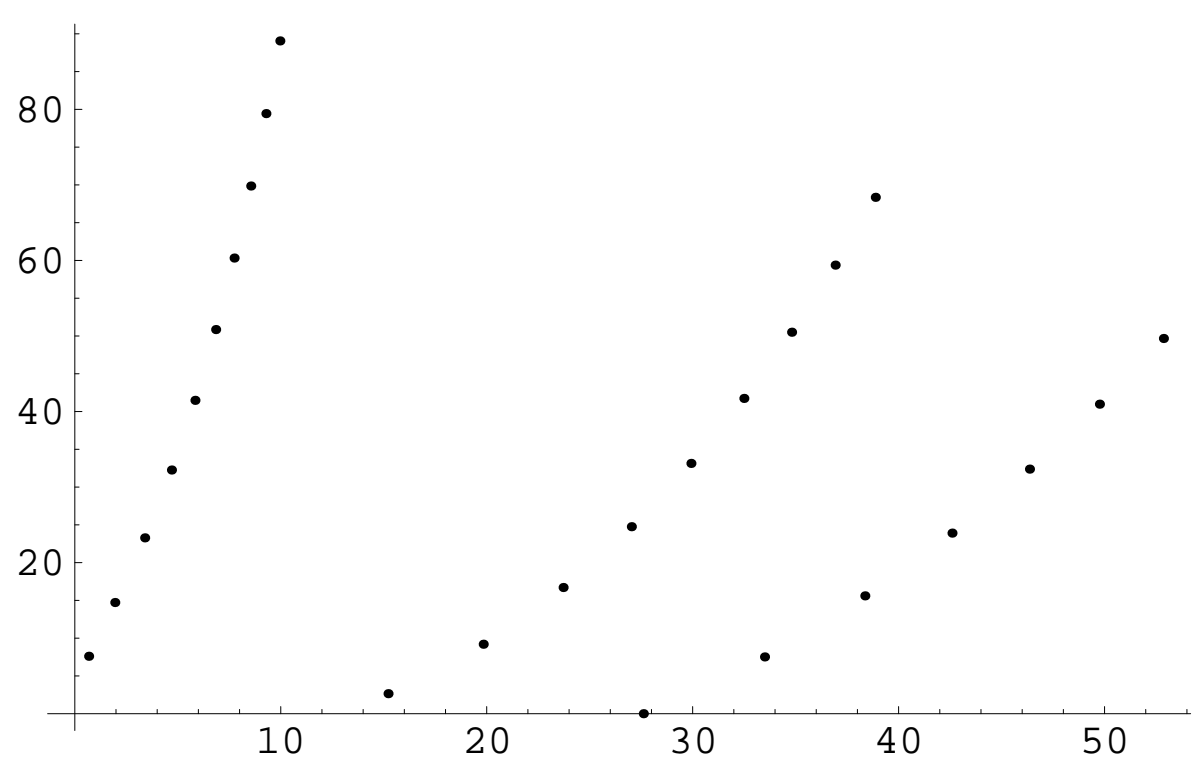

FIG. 2: Evolution in the complex $\mu$-plane of the quasi-normal modes when the mass $M$ varies (with $\alpha=0, z=10$ ). The highest points for each branch correspond to $M \ell=10$. The increment between two adjacent points is $\Delta(M \ell)=1$.

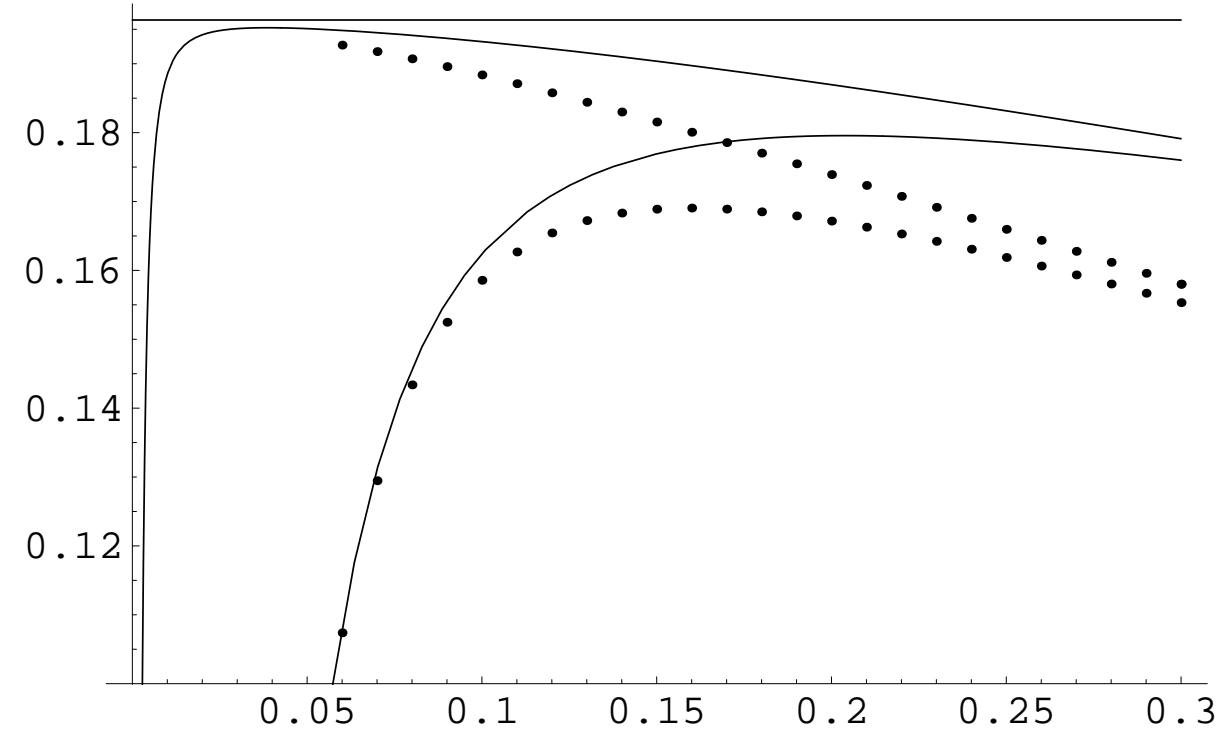

FIG. 3: $-\operatorname{Im}\left[m_{(4)}^{2}\right] /\left(M^{4} \ell^{2}\right)$ as a function of $M \ell(z=50$ for the lower points, $z=1000$ for the upper points). The curves correspond to the analytical estimate (5.7)

continue to approach $\pi / 16$, there is a sudden change in the evolution for $z=50$. This is simply due to the fact that, even if $H \ell \ll 1$, the small values of $M \ell$ become of the same order of magnitude as $H \ell$ and (5.8) is no longer a good approximation: one needs the more general expression (5.7). The two curves in the figure correspond to the analytical estimate given by (5.7) and one sees that the numerical values converge towards these curves for low masses.

Our numerical treatment allows us to continue this quasi-normal branch beyond the regime of validity of the analytical calculations, and in particular for very high masses. Moreover, as mentioned before and as illustrated in Fig. 2] we have found other solutions for $\mu$, away from the real axis, which correspond to additional branches. Each of this branch starts from the real axis after a critical mass threshold has been reached and then evolves away from the real axis as $M \ell$ increases.

Let us now consider the situation when the coupling is allowed to vary. We have plotted in Fig. 目 the evolution of 


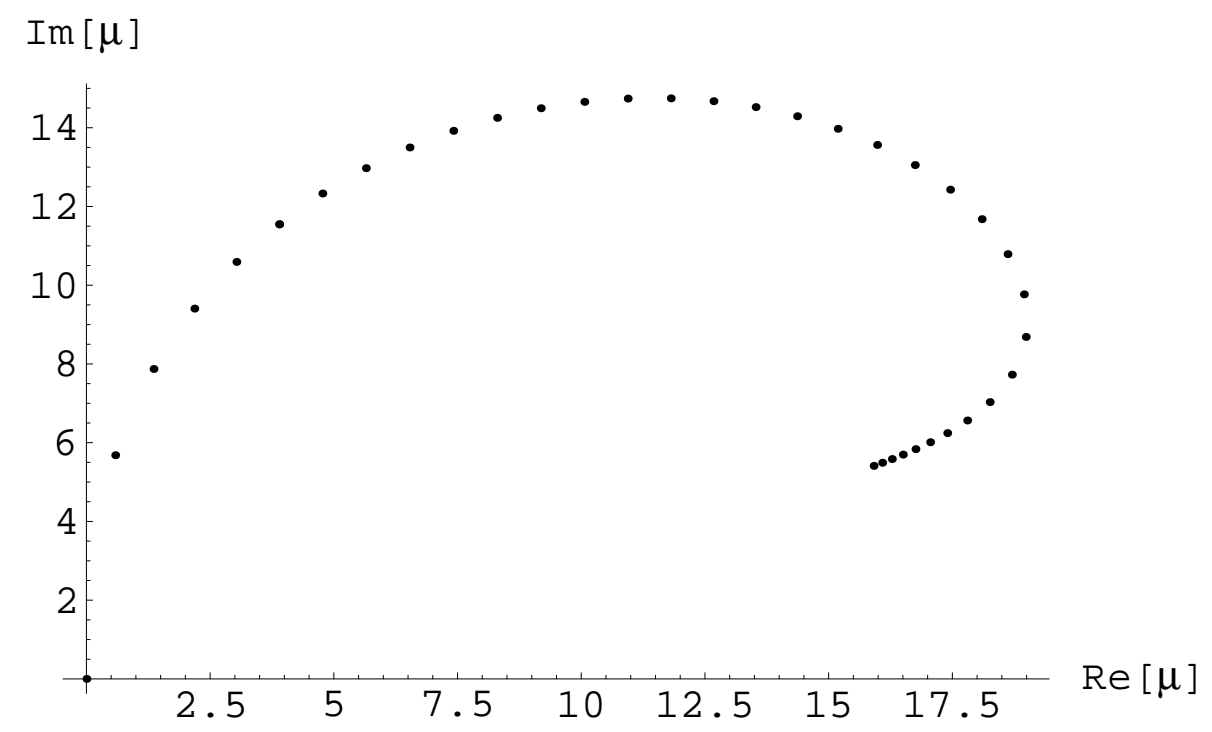

FIG. 4: Evolution in the complex $\mu$-plane of the quasi-normal modes when the coupling varies, in the clockwise direction, from 0 to 7 (with $M \ell=0, z=10$ ). The increment between two points is $\Delta \alpha=0.2$.

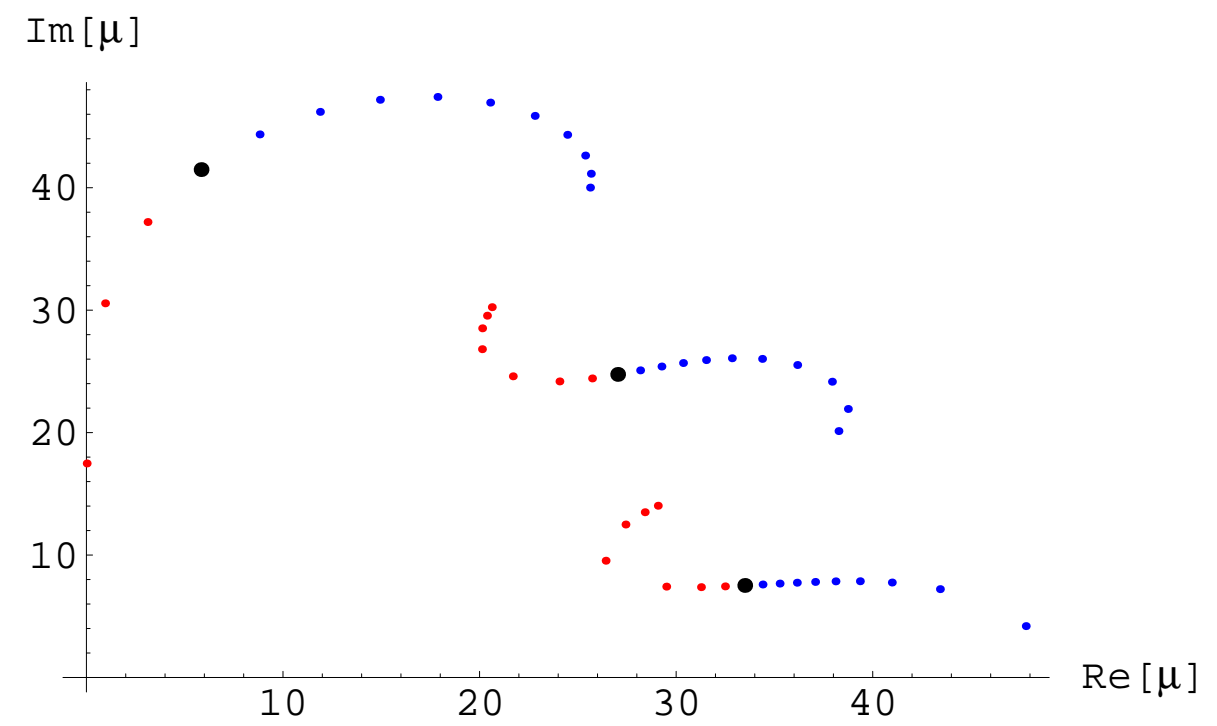

FIG. 5: Variation in the complex $\mu$-plane of the quasi-normal modes when the coupling changes (for $M \ell=5, z=10$ ). The increment between adjacent points is $\Delta \alpha=1$. The three big dots (in black) correspond to the modes for $\alpha=0$. The points on the left of each black dot (in red) correspond to negative couplings and those on the right (in blue) to positive couplings.

the quasi-normal mode with the increasing (positive) coupling in the case $M \ell=0$. What can be observed is that the mode, after going away from the real axis, tends to come back towards it.

In Fig. [5 we have also plotted the same evolution, i.e. with increasing coupling, in the case $M \ell=5$ where several quasi-normal modes are already known to exist in the complex $\mu$-plane for vanishing coupling. We have no analytic expression for this case, but one can observe each of the modes follows a curve analogous to the $M \ell=0$ case. We have also added the evolution corresponding to increasing negative coupling. For the first branch, one finds that the mode converges towards the origin of the complex plane, which could be expected since at some critical value of the coupling one will enter into the localized mode region. For the second branch, one observes that the modes at large negative couplings converge towards the modes of the previous branch at large positive couplings. The same 
behaviour occurs for the third branch.

\section{CONCLUSIONS}

In the present work, we have studied the modes of a massive bulk scalar field that are localized or (quasi-localized) on a brane with a de Sitter geometry, characterized by a Hubble parameter $H$. We have allowed for a coupling between the brane tension and the bulk scalar field, quantified by a dimensionless parameter $\alpha$.

Although the bound state of a massless bulk scalar field without coupling to the de Sitter brane is a zero mode, i.e. its four-dimensional effective mass $m_{(4)}$ vanishes, this is no longer the case for a non-zero bulk mass $M$ or a non zero coupling. One thus finds in general a massive bound state, whose four-dimensional mass depends on $M, \alpha$ and $H$. We have computed explicitly this dependence in limit of a small bulk mass and small coupling.

The mass-squared $m_{(4)}^{2}$ of the bound state is always comprised between zero and $(3 / 2) H^{2}$, value above which one finds a continuum of modes. We have obtained explicitly, for any value of $M$ and $H$, the lower and upper critical values for the coupling corresponding to this range of possible zero modes.

Another approach consists in trying to find a purely four-dimensional description of the scalar field including the effect of the bulk. We have generalized previous such procedures to take into account the coupling, and defined an effective potential for the value on the brane of the scalar field. For small $M$ and $\alpha$, the second derivative of the effective potential yields an effective mass-squared which is in excellent agreement with the rigorous mode approach for small $H$ and in qualitative agreement for large $H$. We have also demonstrated that a class of known exact solutions with a bulk scalar field indeed conforms to the effective potential approach.

Finally, when the three parameters $M, \alpha$ and $H$ are not in the region allowing for bound states, one finds modes whose effective mass has an imaginary part. This means that the corresponding states cannot stay localized on the brane but will escape into the bulk. We have explored numerically the dependence of the real and imaginary part of the four-dimensional mass on the values of the bulk mass and of the coupling and we have given an analytical estimate of the quasi-localized modes for small values of the Hubble parameter, i.e. $H \ell \ll 1$.

\section{Acknowledgments}

We would like to thank K. Koyama for useful communications, in particular, for bringing our attention to exact solutions that conform to the effective potential approach. This work is supported in part by Monbukagaku-sho Grant-in-Aid for Scientific Research (S), No. 14102004. This work was initiated when MS was visiting the gravitation and cosmology group (GReCO) at IAP, Paris. MS is grateful to the Université Paris 7-APC for financial support and to the GReCO members for warm hospitality.

[1] V. A. Rubakov and M. E. Shaposhnikov, Phys. Lett. B 125, 136 (1983); K. Akama, Lect. Notes Phys. 176, 267 (1982) arXiv:hep-th/0001113.

[2] L. Randall and R. Sundrum, Phys. Rev. Lett. 83, 4690 (1999) arXiv:hep-th/9906064.

[3] J. Garriga and M. Sasaki, Phys. Rev. D 62, 043523 (2000) arXiv:hep-th/9912118.

[4] D. Langlois, R. Maartens and D. Wands, Phys. Lett. B 489, 259 (2000) arXiv:hep-th/0006007.

[5] A. V. Frolov and L. Kofman, arXiv:hep-th/0209133

[6] S. L. Dubovsky, V. A. Rubakov and P. G. Tinyakov, Phys. Rev. D 62, 105011 (2000) arXiv:hep-th/0006046.

[7] Y. Himemoto, T. Tanaka and M. Sasaki, Phys. Rev. D 65, 104020 (2002) arXiv:gr-qc/0112027.

[8] N. Sago, Y. Himemoto and M. Sasaki, Phys. Rev. D 65, 024014 (2002) arXiv:gr-qc/0104033.

[9] Y. Himemoto and M. Sasaki, Phys. Rev. D 63, 044015 (2001) arXiv:gr-qc/0010035.

[10] R. Maartens, D. Wands, B. A. Bassett and I. Heard, Phys. Rev. D 62, 041301 (2000) arXiv:hep-ph/9912464.

[11] P. Binetruy, C. Deffayet, U. Ellwanger and D. Langlois, Phys. Lett. B 477, 285 (2000) arXiv:hep-th/9910219.

[12] K. i. Maeda and D. Wands, Phys. Rev. D 62, 124009 (2000) arXiv:hep-th/0008188.

[13] T. Shiromizu, K. i. Maeda and M. Sasaki, Phys. Rev. D 62, 024012 (2000) arXiv:gr-qc/9910076.

[14] D. Langlois, L. Sorbo and M. Rodriguez-Martinez, Phys. Rev. Lett. 89, 171301 (2002) arXiv:hep-th/0206146.

[15] R. G. Cai, J. Y. Ji and K. S. Soh, Phys. Rev. D 57, 6547 (1998) arXiv:gr-qc/9708063.

[16] H. A. Chamblin and H. S. Reall, Nucl. Phys. B 562, 133 (1999) arXiv:hep-th/9903225.

[17] D. Langlois and M. Rodriguez-Martinez, Phys. Rev. D 64, 123507 (2001) arXiv:hep-th/0106245.

[18] D. Langlois, to be published in Prog. Theor. Phys. Suppl. No. 148 arXiv:hep-th/0209261.

[19] K. Koyama and K. Takahashi, arXiv:hep-th/0301165

[20] see e.g., $§ 3.2$ (21) of H. Bateman, "Higher Transcendental Functions, Vol. I" (McGraw-Hill, New York, 1953). 